\title{
Block Copolymers Prepared by Free Radical Polymerization Using $\alpha$-Methylstyrene-containing Precopolymer as Macroinitiator
}

\author{
By Shan JIANG, Jianping DENG, and Wantai YANG*
}

A novel way to prepare block copolymers is presented. Copolymerizations of $\alpha$-methylstyrene (AMS) and glycidyl methacrylate (GMA) were carried out. The resulting copolymers, designated as poly(AMS-co-GMA) (PAG), were characterized by Fourier transform infrared spectroscopy (FT/IR), gel permeation chromatography (GPC), ${ }^{1} \mathrm{H}$ NMR and thermogravimetric analyses (TGA). Based on TGA, two main degradations with two inflection points were observed at $178.20^{\circ} \mathrm{C}$ and $342.1{ }^{\circ} \mathrm{C}$ in PAG, while only one degradation with an inflection point at $305.7^{\circ} \mathrm{C}$ on homopolymer of GMA (PGMA), indicating the depolymerization started with scission of weak bonds in PAG. When heated, the scission of those weak bonds gave birth to chain radicals, which could further initiate the polymerization of other monomers. On the basis of this finding, a series of block copolymers, PAG-block-PMMA $\left(M_{\mathrm{n}}=63100 \mathrm{~g} / \mathrm{mol}, M_{\mathrm{w}} / M_{\mathrm{n}}=1.77\right)$ and PAG-block-PSt $\left(M_{\mathrm{n}}=44700 \mathrm{~g} / \mathrm{mol}, M_{\mathrm{w}} / M_{\mathrm{n}}=1.63\right)$, were prepared by simply heating PAG in the presence of the second monomer. This simple method is expected to be applied to kinds of monomers, and it might be suitable even for industrial applications.

KEY WORDS: Block Copolymer Synthesis / Radical Polymerization / $a$-Methylstyrene / Macroinitiator /

The polymerization of monomers using macroinitiators containing both appropriate polymer segments and initiating moieties provides an opportunity for the synthesis of a variety of well-defined block and graft copolymers. Since controlled polymerizations initiated by living macroinitiators ${ }^{1-5}$ have to be carried out under strict conditions, other macroinitiators were used instead, among which polymers containing scissile azo groups and peroxy groups have been well studied. ${ }^{6-15}$ These prepolymers decompose into free radicals, which can initiate the polymerization of other monomers by free radical mechanisms to yield block copolymers, ${ }^{6-14}$ or react with the double bonds present in the polymer main chains to provide graft copolymers. ${ }^{6,15}$

AMS is commercially available, and without known toxicity. Nevertheless, AMS is reluctant to undergo radical homopolymerization under usual conditions due to the steric hindrance and low ceiling temperature. On the other hand, copolymerizations of AMS and other monomers have been extensively investigated. ${ }^{16-18}$ The formation of stable radicals in AMS and the depropagation reactions can hinder the polymerization process, by which the copolymerization rate is sharply reduced. ${ }^{16,17}$ The primary advantage associated with this rate control is that low molecular weight polymers can be produced without the requirement for large quantities of chain transfer agents or initiators. ${ }^{18,19}$

Recently, we found that when an oligomer (PAG) consisting of AMS and GMA was melt blended with polypropylene (PP), the GMA units in the oligomer could be successfully grafted onto the PP chain. At the melting temperature, the oligomer was supposed to depolymerize to form short-chain free radicals with reactive functional groups, and in turn the free radicals enabled the subsequent grafting reaction of the short chains onto the polymer substrates by free radical coupling reactions. $^{20}$ Since this strategy is based on a free radical mechanism, it is certainly feasible to initiate other monomers to polymerize, and the products are expected to be block copolymers.

This paper reports on the design and synthesis of block copolymers on the basis of the above idea. We found that methyl methacrylate (MMA) and styrene (St) smoothly underwent polymerization, initiated by the macroinitiator PAG obtained in advance. The results demonstrated that it is possible to extend the range of monomers for the preparation of AMS-containing polymeric initiators and multiblock copolymers based on the idea developed in the present article. This AMS based technique is convenient, efficient, and can be applied to a lot of monomers to prepare highly functional copolymers with unique structures.

\section{EXPERIMENTAL}

\section{Materials}

$\alpha$-Methylstyrene (AMS, Alfa Aesar) and glycidyl methacrylate (GMA, Aldrich) were distilled under vacuum. Methyl methacrylate (MMA) and styrene (St) were purchased from Beijing Chemical Reagent Corp. and were distilled under reduced pressure before use. $a, a^{\prime}$-Azobisisobutyronitrile (AIBN) was purchased from Beijing Chemical Reagent Corp. It was recrystallized from absolute ethanol, dried under vacuum at room temperature and stored in an amber bottle. Tetrahydrofuran (THF), toluene, methanol and $N, N$-dimethylformamide (DMF) were purchased from Beijing Chemical Reagent Corp. and used as received. 


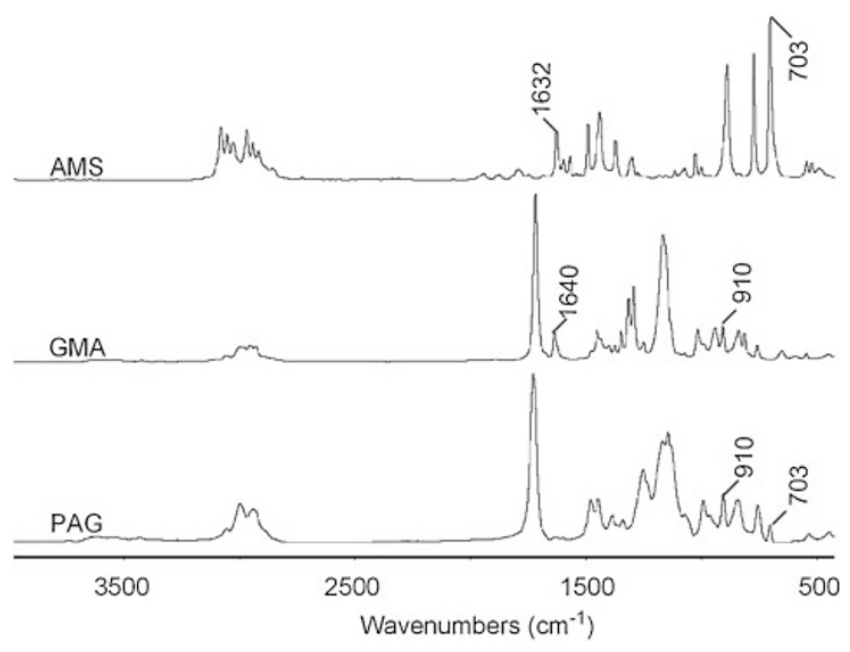

Figure 1. FT/IR spectra of AMS, GMA and poly(AMS-co-GMA).

\section{Instruments}

FT/IR spectra of polymers were recorded with Hitachi 27050 infrared spectrometer on solid samples as $\mathrm{KBr}$ pellets. ${ }^{1} \mathrm{H}$ NMR spectra were obtained on a Bruker AV600 spectrometer in $\mathrm{CDCl}_{3}$ at room temperature. ${ }^{1} \mathrm{H}$ chemical shifts were referenced to tetramethyl silicane (TMS) with the residual nondeuterated solvent signal at $\delta=7.23 \mathrm{ppm}$. Molecular weights and molecular weight polydispersities $\left(M_{\mathrm{w}} / M_{\mathrm{n}}\right)$ of the copolymers were determined by GPC (Waters 515-2410 system) calibrated by using polystyrenes as standards and THF as an eluent. TGA was performed on a NETZSCH TG 209 instrument on powder samples at a heating rate of $10^{\circ} \mathrm{C} / \mathrm{min}$ under nitrogen atmosphere from 25 to $500^{\circ} \mathrm{C}$, and a NETZSCH STA $449 \mathrm{C}$ instrument at $260^{\circ} \mathrm{C}$ under nitrogen atmosphere, respectively.

Synthesis of Poly( $\alpha$-methylstyrene-co-glycidyl methacrylate) (PAG) Macroinitiator

Copolymerization of AMS and GMA was carried out at $65^{\circ} \mathrm{C}$ in THF under stirring using AIBN as initiator, in a previously argon-purged glass reactor. After $16 \mathrm{~h}$ copolymerization, the copolymer PAG was recovered by precipitation from a large excess of methanol. To remove the residual monomer, the crude product was further purified by repeatedly dissolving in THF and reprecipitating by adding methanol three times. Finally, the white products were dried in a vacuum oven at $50{ }^{\circ} \mathrm{C}$ for $24 \mathrm{~h}$ and stored in a desiccator. The products were analyzed by FT/IR spectroscopy (KBr tablet). The conversion was determined gravimetrically. The composition of the obtained copolymers was analyzed by means of ${ }^{1} \mathrm{H}$ NMR spectroscopy.

Preparation of Poly(glycidyl methacrylate) (PGMA) Reference Polymer

The synthesis and purification of the PGMA referencepolymer followed the same procedure as described above but without AMS.

\section{Synthesis of Block Copolymers}

The synthesis of block copolymers were achieved by using PAG obtained above as macroinitiator. PAG and a second monomer were placed in the reactors, and heated at $80-120{ }^{\circ} \mathrm{C}$ in bulk and solution under Ar atmosphere. Samples were taken by a syringe during the polymerization, precipitated from methanol and dried in a vacuum oven. Yields were determined gravimetrically. GPC measurements demonstrate the formation of a new polymer at higher molecular weight next to the dead polymers occurring at lower molecular weight.

\section{RESULTS AND DISCUSSION}

\section{Synthesis and Characterization of PAG Macroinitiators}

GMA was polymerized in THF with AIBN as the initiator and different amounts of AMS at $65^{\circ} \mathrm{C}$. The polymerization rate was determined with and without AMS, respectively. The FT/IR spectra of AMS, GMA and PAG are given in Figure 1, which shows the double bond peaks at $1632 \mathrm{~cm}^{-1}$ and 1640 $\mathrm{cm}^{-1}$ disappeared, while the phenyl band at $703 \mathrm{~cm}^{-1}$ and glycidyl band at $910 \mathrm{~cm}^{-1}$ remained after polymerization. It thus demonstrated the formation of PAG copolymers.

Monomer conversions were determined gravimetrically. Figure 2 shows that the polymerization rate decreases in the presence of AMS, which is caused by the formation of stable radicals in AMS and the depropagation reactions. Without AMS, the monomer conversion increases quickly during the first $4 \mathrm{~h}$, and then turns to be constant. With AMS, the polymerization rate increases linearly in $8 \mathrm{~h}$. The composition of the copolymers was determined according to ${ }^{1} \mathrm{H}$ NMR spectra, as shown in Figure 3. The molar fraction $\mathrm{F}_{1}$ of AMS $\left(\mathrm{M}_{1}\right)$ in the copolymer could be determined by comparing the intensity of the signals assigned to the aromatic hydrogens in AMS to the integrated signal intensity of $-\mathrm{COO}-\mathrm{CH}_{2}$ - in the GMA units. The relevant results are presented in Table I.

From Table I, $\mathrm{F}_{1}$ is lower than the corresponding molar fraction $f_{1}$ in feed, which indicates that GMA was consumed 


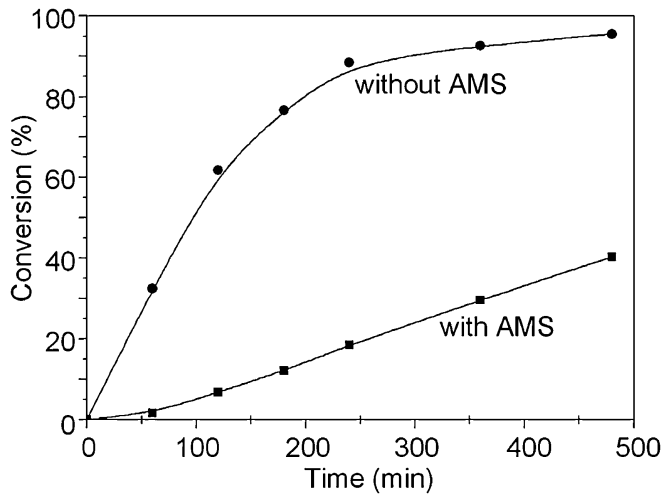

Figure 2. Dependence of monomer conversion as function of time in the polymerization of $\mathrm{GMA}$ with $\mathrm{AMS}(\mathrm{AMS} / \mathrm{GMA}=1 / 2$ (mol/mol); $\mathrm{AIBN} /$ $(A M S+G M A)=4 \%(w / w))$ and without AMS $($ AIBN/GMA $=4 \%(w / w))$. The polymerization temperature was $65^{\circ} \mathrm{C}$.

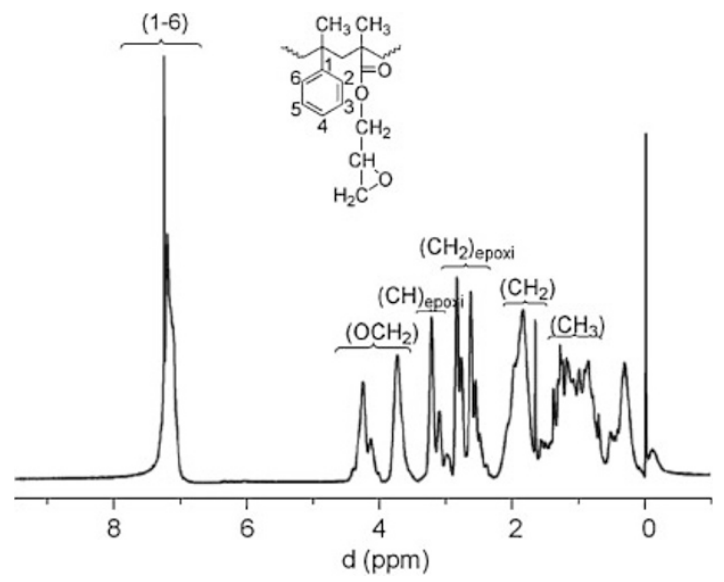

Figure 3. ${ }^{1} \mathrm{H}$ NMR spectra of poly(GMA-co-AMS) in $\mathrm{CDCl} 3$ at room temperature.

Table I. Polymerization Data of AMS and GMA in $\mathrm{THF}$ at $65^{\circ} \mathrm{C}\left([\mathrm{AIBN}]=5.58 \times 10^{-2} \mathrm{M}\right)$

\begin{tabular}{cccccc}
\hline run & $\mathrm{f}_{1}$ & AIBN $(\mathrm{M})$ & $\mathrm{F}_{1}{ }^{\mathrm{a}}$ & $M_{\mathrm{n}}{ }^{\mathrm{b}}(\mathrm{g} / \mathrm{mol})$ & $\left(M_{\mathrm{w}} / M_{\mathrm{n}}\right)^{\mathrm{b}}$ \\
\hline PAG-1 & 0.333 & $5.58 \times 10^{-2}$ & 0.225 & 3100 & 1.26 \\
PAG-2 & 0.111 & $5.58 \times 10^{-2}$ & 0.078 & 4500 & 1.45 \\
PAG-3 & 0.059 & $5.58 \times 10^{-2}$ & 0.025 & 6500 & 1.48 \\
PAG-4 & 0.333 & $0.70 \times 10^{-2}$ & 0.199 & 5400 & 1.36 \\
PAG-5 & 0.111 & $0.70 \times 10^{-2}$ & 0.038 & 9400 & 1.67 \\
PGMA & $/$ & $/$ & $/$ & 12100 & 2.81 \\
\hline
\end{tabular}

${ }^{a}$ values measured by ${ }^{1} \mathrm{H}$ NMR, referenced to TMS. ${ }^{b}$ values measured by GPC, calibrated with polystyrene standards.

more rapidly than AMS and the GMA ratio in the monomer mixture progressively decreased. Table I also shows the presence of AMS influences the molecular weight of the resulting polymers. The number-average molecular weight $\left(M_{\mathrm{n}}\right)$ and molecular weight distribution $\left(M_{\mathrm{w}} / M_{\mathrm{n}}\right)$ of the copolymers reduce with f1 increasing. The $M_{\mathrm{n}}$ of the copolymer, obtained with $\mathrm{f}_{1}=0.333$ and $\operatorname{AIBN}=5.58 \times 10^{-2} \mathrm{M}$, is 3100 with a fairly narrow molecular weight distribution, $M_{\mathrm{w}} / M_{\mathrm{n}}=1.26$. These values are clearly lower than those observed in common radical polymerization. Since AMS causes a drastic decrease in both the rate of polymerization<smiles>C/C=C(/C)c1ccccc1</smiles><smiles>CCC(C)c1ccccc1</smiles><smiles>CCCC(C)(CC)C(C)(CC)c1ccccc1</smiles><smiles>CCC(C)(C)C(C)(CC)C(=O)OCC1CO1</smiles>

Scheme 1. Possible structures of most AMS units in the PAGs.

and the average degree of polymerization, a conclusion can be drawn that the kinetic chain with a terminal AMS-radical would rather terminate with another radical or transfer to a monomer than propagate. With regard to the structure of these copolymers, it can be concluded that most AMS is either present as an end group (as a result of chain transfer or termination by disproportionation) or within the chain as a unit of a head-to-head structure (as a result of termination by recombination) (as illustrated in Scheme 1). The chemical analysis of the PAG block does not reveal any exotic structure (Figure 3).

\section{Thermal Decomposition of PAG Macroinitiator}

Thermogravimetric (TG) traces for PGMA, PAG-4 $\left(M_{\mathrm{n}}=\right.$ $\left.6200, \mathrm{~F}_{\mathrm{AMS}}=0.199\right)$ and PAG-5 $\left(M_{\mathrm{n}}=9400, \mathrm{~F}_{\mathrm{AMS}}=0.038\right)$ at heating rate $10^{\circ} \mathrm{C} / \mathrm{min}$ under nitrogen are shown in Figure 4. In the case of homopolymer PGMA, only one step is found in the TG curve, with an inflection point at $305.7^{\circ} \mathrm{C}$, corresponding to the degradation by random scission of the polymer main chain. PGMA is structurally similar to poly(methyl methacrylate) (PMMA). Both have monomer units containing quaternary backbone carbons, which are alternating with secondary backbone carbons. The difference lies in their substitutent groups. Like PMMA, ${ }^{21}$ PGMA should thermally degrade mainly by random scission depolymerization. It is found that PAGs degrade in a two-step in the TG measurement, with two inflection points at $178.2^{\circ} \mathrm{C}$ and $342.1^{\circ} \mathrm{C}$ (Figure 4), which indicates presence of weak links in the PAG sample. At lower temperature, the weakest bonds, most probably exist in the sequences of AMS units, seem to be scissored firstly. ${ }^{22}$

As the AMS content increases in the copolymer, the onset temperature of the degradation shifts to the lower temperature side, as seen in Figure 4. This fact could be explained as 


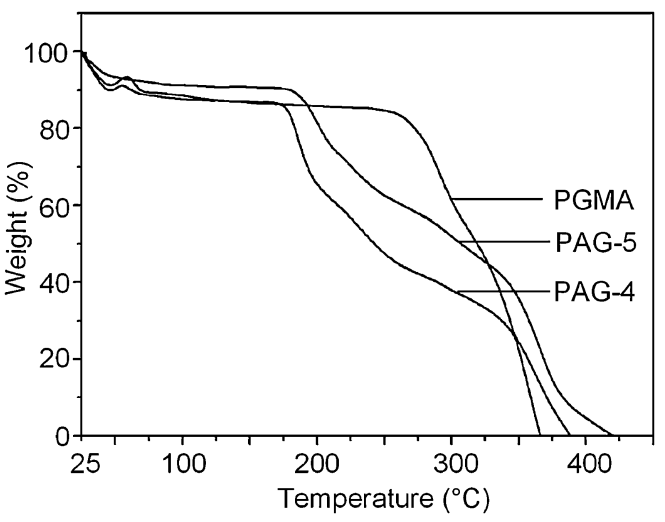

Figure 4. TGA curves of PAG-4, PAG-5 and PGMA (temperature range: 25$450^{\circ} \mathrm{C}$; rate: $10^{\circ} \mathrm{C} / \mathrm{min} ; \mathrm{N}_{2}$ atmosphere).

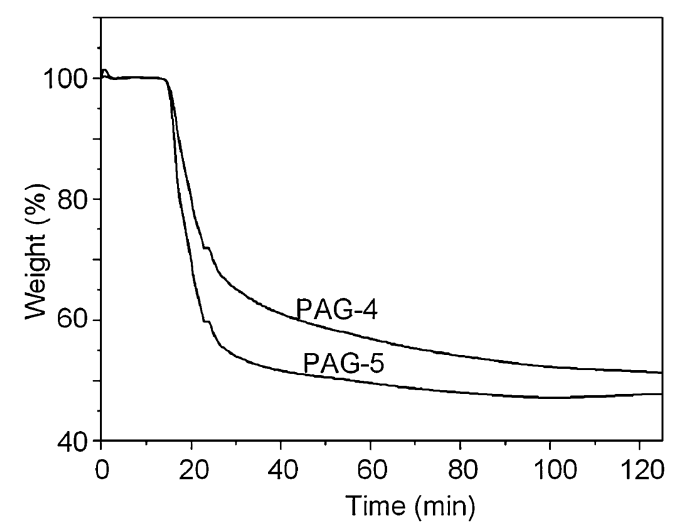

Figure 5. Time dependence of weight loss in isothermal degradation of PAGs (Temperature: $260^{\circ} \mathrm{C}$; time: $2 \mathrm{~h} ; \mathrm{N}_{2}$ atmosphere).

follows: The thermal cleaving reaction of the weakest bonds, which increase as AMS moiety increases, gives birth to a radical species, and then initiates decomposition of the PAGs.

Same PAG samples were heated at $10^{\circ} \mathrm{C} / \mathrm{min}$ up to $260^{\circ} \mathrm{C}$ under nitrogen, and then maintained at $260^{\circ} \mathrm{C}$ for another $2 \mathrm{~h}$. As shown in Figure 5, the weight loss of PAG samples turns to be constant in short time, respectively. The final weight loss increases as the AMS moiety in PAG increases, which further demonstrates that weak bonds exist in the AMS sequences, and the thermal degradation should be based on a free-radical mechanism. The result offers a theory base for the following experiments, in which AMS-containing precopolymer is used as a macroinitiator to prepare block copolymers. Block copolymers synthesized with PAG macroinitiator.

\section{Synthesis of PAG-b-PMMA in Bulk and Solution}

Poly(AMS-co-GMA) $\left(M_{\mathrm{n}}=2900 \mathrm{~g} / \mathrm{mol}, M_{\mathrm{w}} / M_{\mathrm{n}}=1.37\right)$ was heated in the presence of MMA at $90{ }^{\circ} \mathrm{C}$ without additional initiator in bulk. Figure 6 shows that monomer conversion increase with time linearly, and reach $15 \%$ in $1.5 \mathrm{~h}$. It should be pointed out that no polymerization of MMA occurred without adding PAG with other conditions kept the same. Since no additional initiator was added, the polymerization only could be initiated by the PAG, and the product should be block

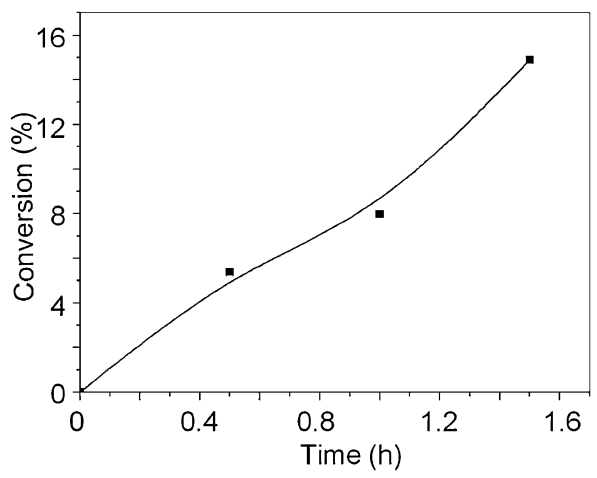

Figure 6. Dependence of monomer conversion as function of time in the bulk polymerization of MMA initiated by PAG precursor (PAG/ MMA $\left.=1 / 2800(\mathrm{~mol} / \mathrm{mol}) ; T=90^{\circ} \mathrm{C}\right)$.

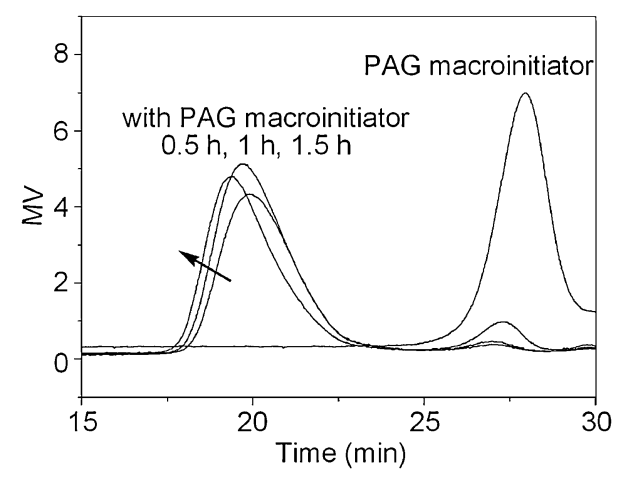

Figure 7. GPC curves (THF as eluent) for original PAG and samples obtained during bulk polymerization of MMA using PAG macrointitor $\left(\mathrm{PAG} / \mathrm{MMA}=1 / 2800(\mathrm{~mol} / \mathrm{mol}) ; T=90^{\circ} \mathrm{C}\right)$.

copolymer. From the GPC spectra (Figure 7), the molecular weight of the new copolymer, resulted from the reinitiation, is larger than that of the original polymer PAG. The molecular weight of block copolymer almost keeps constant during the polymerization, which is consistent with conventional free radical polymerization mechanism. Due to the gel effect, the molecular weight distribution increases from 1.89 to 2.15 , while the conversion increases from $5.4 \%$ to $15.0 \%$.

A series of polymerizations of MMA in the presence of different PAGs were also carried out in bulk. From Table II, under the same conditions, the conversion of MMA reduces as the molar fraction of AMS in the copolymer reduces. In contrast to the conversion, the molecular weight of block polymer increases when the molar fraction of AMS in the copolymer gets reduced. It agrees well with the above assumption that the polymerization occurred at the AMS sites. In Table II, it is noticed that the molecular weights measured

Table II. Bulk Polymerization Data of MMA Initiated by Different PAGs (PAG/MMA $=0.01 \mathrm{~g} / \mathrm{ml}, T=90^{\circ} \mathrm{C}$, time $=90 \mathrm{~min}$ )

\begin{tabular}{cccc}
\hline Precursor polymer & Conversion $(\%)$ & $M_{\mathrm{n}}$ & $M_{\mathrm{w}} / M_{\mathrm{n}}$ \\
\hline PAG-1 $\left(\mathrm{F}_{\mathrm{AMS}}=0.225\right)$ & 17.4 & 368000 & 2.15 \\
PAG-2 $\left(\mathrm{F}_{\mathrm{AMS}}=0.078\right)$ & 6.2 & 430000 & 1.71 \\
PAG-3 $\left(\mathrm{F}_{\mathrm{AMS}}=0.025\right)$ & 4.3 & 521000 & 1.55
\end{tabular}


by GPC are large $(368000-521000 \mathrm{~g} / \mathrm{mol})$, which should be caused by the occurrence of some pre-gel component. The polydispersity of the final product, obtained from bulk polymerizations, becomes much wider as the conversion increases, which should also be caused by the gel effect.

Solution polymerizations of MMA in the presence of PAG were respectively carried out in butanone and toluene, without additional initiator. The results are consistent with those of the bulk polymerizations above. A series of experiments carried out at different temperatures showed that no polymerization of MMA occurred in $8 \mathrm{~h}$ at $70^{\circ} \mathrm{C}$, while the percent conversion reached $59.6 \%$ in $8 \mathrm{~h}$ at $90^{\circ} \mathrm{C}$. This demonstrated that the radicals initiating the second-step polymerizations should be formed at elevated temperatures. With the ratio of PAG to monomer kept constant, experiments with different concentrations of monomer were carried out in toluene. It turns out that with the monomer concentration increasing, both the yield and the molecular weight of product increase. With $[\mathrm{MMA}]=0.944 \mathrm{M}$, the percent conversion reached $20.7 \%$ in $5 \mathrm{~h}$ and the $M_{\mathrm{n}}$ of obtained polymer is 23200. With $[\mathrm{MMA}]=3.776 \mathrm{M}$, the percent conversion reached $66.5 \%$ in $5 \mathrm{~h}$, and the $M_{\mathrm{n}}$ is 60100 . All these phenomena strongly demonstrate that the polymerization of MMA initiated by PAG macroinitiator is a conventional free radical process.

\section{Synthesis of PAG-b-PSt in Solution}

St was also used as the second polymer. The polymerization of St initiated by PAG was carried out in toluene at $100{ }^{\circ} \mathrm{C}$. The percent conversion of $\mathrm{St}$ also linearly increases as time prolongs. It was proved by experiment that no polymerization occurred without PAG with other conditions the same. The change of $M_{\mathrm{n}}$ of the block copolymer is presented in Figure 8. The remaining PAG peaks are observed, indicating the presence of polymer chains without special AMS sites, which are supposed to reinitiate polymerizations, and products from side reactions (such as recombination of two GMA-ended macroradicals coming from the thermal decomposition of prepolymer). From Figure 7, the remaining PAG peaks have also been observed when PAG was used to initiate the polymerization of MMA at $90{ }^{\circ} \mathrm{C}$.

However, for the two monomers MMA and St, difference could be observed by comparing Figures 7 and 8. In the GPC curves for the polymerization of MMA carried out at $90^{\circ} \mathrm{C}$, the remaining $\mathrm{PAG}$ peaks shift to higher molecular weight (Figure 7), compared with the original PAG peak. This phenomenon demonstrates that the unreacted PAG chains and side products own higher $M_{\mathrm{n}}$ than those PAG chains that might act as macroinitiators. However, the GPC curves for the reinitiation of St carried out at $100{ }^{\circ} \mathrm{C}$ show the remaining $\mathrm{PAG}$ peaks shift to lower molecular weight (Figure 8), indicating depolymerization of original PAG took place during the reinitiation. Moreover, the depolymerization must be promoted a lot by the enhanced reaction temperature.

The nature of the reinitiating mechanism is not yet revealed. The proposed mechanism starts from thermal decomposition of the head-to-head bond between an AMS unit and a GMA unit

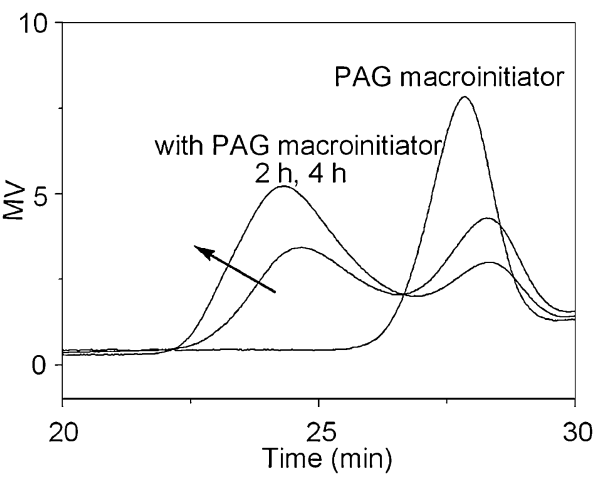

Figure 8. GPC curves (THF as eluent) for original PAG and samples obtained during solution polymerization of St using PAG macrointitor (PAG/St $=1 / 270(\mathrm{~mol} / \mathrm{mol})$; solvent: toluene; $\left.T=100^{\circ} \mathrm{C}\right)$.

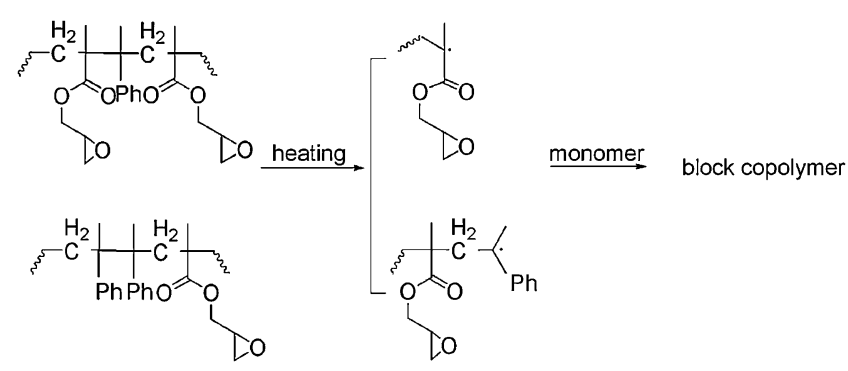

Scheme 2. The proposed mechanism of the block copolymerization initiated by PAG macroinitiator.

or two AMS units in the PAG oligomer, which is formed after combination termination during the first step polymerization. The radical thus formed initiates chain growth by conventional free radical polymerization, as shown in Scheme 2. PAG was also applied for the synthesis of other block copolymers, such as PAG-block-poly( $N$-vinyl pyrrolidone), PAG-block-poly(methyl acrylate), and PAG-block-poly(hydroxyethyl methacrylate). Apart from PAG, other AMS-containing macroinitiators were also designed and synthesized, such as poly(AMS-coMMA) (PAM), by which PAM-block-PSt were synthesized.

\section{CONCLUSION}

A new approach to obtain block copolymer was presented. In the presence of a second monomer, heating of the oligomers derived from AMS leads to block copolymers. The polydispersities of all the prepolymers and block copolymers are narrow. This new system might be suitable even for industrial application because of its versatility and simplicity combined with mild conditions and short reaction times. Further investigations are focused on the determination of the mechanism of reinitiation and the optimization of the reaction conditions for different monomers.

Acknowledgment. We gratefully thank the National Science Foundation of China (20574004), Special Research Fund for the Doctoral Program of Higher Education (20060010001) and Major Project for Polymer Chemistry and Physics Subject 
Construction from Beijing Municipal Education Commission (BMEC) (XK 100100540, XK 100100640) for financial support of this work.

Received: October 29, 2007 Accepted: February 22, 2008 Published: April 16, 2008

\section{REFERENCES}

1. S. H. Qin, K. Y. Qiu, G. Swift, D. G. Westmoreland, and S. Wu, J. Polym. Sci., Part A: Polym. Chem., 37, 4610 (1999).

2. B. de Boer, U. Stalmach, P. F. van Hutten, C. Melzer, V. V. Krasnikov, and G. Hadziioannou, Polymer, 42, 9097 (2001).

3. R. Krishnan, K. Sabdham, and V. Srinivasan, Eur. Polym. J., 39, 205 (2003).

4. K. Ishizu, R. A. Khan, Y. Ohta, and M. Furo, J. Polym. Sci., Part A: Polym. Chem., 42, 76 (2004).

5. G. Hild and J. P. Lamps, Polymer, 39, 2637 (1998).

6. E. E. Hamurcu, B. Hazer, Z. Misirli, and B. M. Baysal, J. Appl. Polym. Sci., 62, 1415 (1996).

7. M. S. Eroğlu, B. Hazer, O. Güven, and B. M. Baysal, J. Appl. Polym. Sci., 60, 2141 (1996).
8. B. Hazer, Makromol. Chem., 193, 1081 (1992).

9. J. M. G. Cowie and Y. P. Mehrdad, Bri. Polym. J., 16, 127 (1984).

10. Y. Haneda, H. Terada, M. Yoshida, A. Ueda, and S. Nagai, J. Polym. Sci., Part A: Polym. Chem., 32, 2641 (1994).

11. C. I. Simionescu, G. David, A. Ioanid, V. Paraschiv, G. Riess, and B. C. Simionescu, J. Polym. Sci., Part A: Polym. Chem., 32, 3123 (1994).

12. I. Piirma and L. P. Chou, J. Appl. Polym. Sci., 24, 2051 (1979).

13. B. Hazer, A. Ayas, N. Besirli, and N. Saltek, Makromol. Chem., 190, 1987 (1989).

14. J. J. Laverty and Z. G. Gardlund, J. Polym. Sci., Polym. Chem. Ed., 15, 2001 (1977).

15. B. Hazer, Macromol. Chem. Phys., 196, 1945 (1995).

16. R. F. Nogueira and M. I. B. Tavares, J. Appl. Polym. Sci., 88, 1004 (2003).

17. M. Ueda, M. Mano, H. Mori, and H. Ito, J. Polym. Sci., Part A: Polym. Chem., 29, 1779 (1991).

18. D. Kukulj and T. P. Davis, Macromolecules, 31, 5668 (1998).

19. C. Barner-Kowollik and T. P. Davis, Macromol. Theory Simul., 10, 255 (2001).

20. J. P. Deng, S. J. Liang, C. R. Zhang, and W. T. Yang, Macromol. Rapid Commun., 28, 2163 (2007).

21. Y. M. Lee and D. S. Viswanath, Polym. Eng. Sci., 40, 2332 (2000).

22. Y. Shibasaki, M. Yang, and L. Sun, Polym. Adv. Technol., 13, 260 (2002). 